\title{
Expanding the use of digital technology across the NHS
}

\author{
Emeritus Professor Alan Glasper, from the University of Southampton, discusses the Government's ambition \\ to roll out advances in digital healthcare technology to improve patient care in the NHS
}

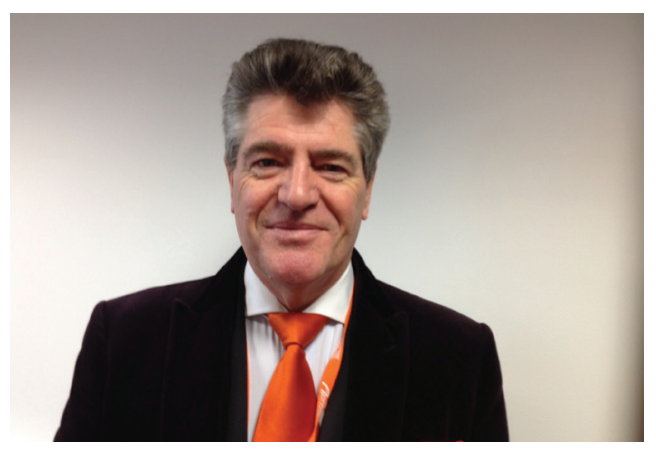

n September 2016, Secretary of State for Health Jeremy Hunt MP announced that he was going to award substantial funding to a range of NHS trusts to help them develop excellence in harnessing digital technology for the improvement of patient care (Department of Health (DH), 2016a).

These new plans to fast-track digital excellence and improve the digital skills of the NHS workforce are part of a major drive to expand the use of digital technology across the NHS. This initiative is a direct response to the findings of the Wachter review of NHS technology, entitled Making IT Work: Harnessing the Power of Health Information Technology to Improve Care in England (DH, 2016b).

\section{Background}

Professor Robert Wachter is internationally renowned for his application of technology to medical care. He is also an expert in the field of medical errors and patient safety. His book The Digital Doctor: Hope, Hype, and Harm at the Dawn of Medicine's Computer Age (Wachter, 2015) argues that computerised medicine can only expand and ultimately improve health. Wachter and his report team are adamant that for the NHS to continue to provide a high level of affordable health care, it has to adapt to changes in healthcare delivery via digital technology. This transformation will involve sweeping changes in how staff view this technology and, importantly, how it affects the NHS workforce and staff training.
However, Professor Wachter is just one of many health professionals in the history of medicine who have endeavoured to harness the power of information technology, not least telemedicine which can trace its origins back to the late 19th century. For example, the first recorded telephone consultation in the UK took place in 1879 to discount a provisional diagnosis of croup (Anonymous, 1879). Later, the use of telephone triage became commonplace and was the basis of a number of nurse-led initiatives that ultimately led to the development of NHS Direct and its later replacement with the national helpline 111. (Glasper, 1993). Similarly, SMS text messaging has been widely used throughout the health service, with Hasvold et al (2011) showing that the sending of SMS reminders to outpatient attendees improved DNA (did not attend) rates. Indeed, it has been argued that telemedicine offers health professionals opportunities to address social and geographical inequalities in healthcare delivery (May and Ellis, 2001).

In contemporary health care, Rosengren et al (2011) have described how a new model of 'teleoncology' care in Australia has enabled rural and indigenous patients with cancer to receive specialist consultations and chemotherapy in their own rural communities, thereby reducing access difficulties. Indeed, during a recent visit to Orkney as part of a Nursing and Midwifery Council (NMC) monitoring exercise, I witnessed telemedicine first hand when a consultant neurological specialist based in Aberdeen carried out an assessment of a patient using both visual and auditory technology where he and the patient could see and talk to each other in real time.

\section{Government plans to expand the use of healthcare technology}

Knowledge transfer lies at the heart of this new government initiative to harness the power of digital technology to make improvements in health care. To achieve this, it will invite universities to host a new NHS digital academy with the aim of driving forward advances in digital health technology. Links to such university departments will be essential in facilitating the training of NHS professionals in the key fundamental skills they will need to deliver the next generation of digital healthcare. It is intended that the new digital academy will work closely with 12 selected NHS trusts that will be at the vanguard of these developments as 'digitisation pioneers'.

This will be achievable through technology grants of up to $£ 10$ million to each trust with an expectation that they use the money wisely to develop and deliver new pioneering approaches to health care by fully engaging with digital technology advances. In addition to the investment in in technology and infrastructure, the funding will be used to deliver staff training to develop a cadre of new generation chief clinical information officers, who in turn will develop innovations in digital healthcare technology.

Importantly, the selected trusts, which include University Hospitals Southampton NHS Foundation Trust and City Hospitals Sunderland NHS Foundation Trust, will help other trusts to emulate their achievements in harnessing the power of digital technology. Furthermore, the Government is anticipating that these digital vanguard trusts will enter into partnership with an international organisation of their choice to expand their use of digital technology knowledge in the pursuit of excellence in care delivery.

Jeremy Hunt has indicated that additional funding will be given to a further $20 \mathrm{NHS}$ trusts to avail themselves of coaching from the envisaged NHS digital academy, with , 5 million each to roll out innovations in digital technology across their organisations. In responding to the Wachter review, Jeremy Hunt is endorsing its findings, which make it clear that innovations in digital technology are only as good as the health professional using 
it, so people will need to develop the skills and expertise to deliver new digitalised services.

\section{Establishing new digital technology to enhance patient care}

The Government has a range of digital technology measures that it will develop to enhance overall patient care. These include:

- Innovations in primary care. One of the first services to be targeted will be primary care and new technology will enable patients to register with a GP, access healthcare records and receive medical advice and information via their computer, tablet or smartphone. It is intended that these new services will be available from the end of 2017 and are targeted at the increasing number of people who are using smartphones and other mobile devices to access public services. The Government hopes that this will make the NHS easier to use.

- Expanding the 111 non-emergency telephone service. This service, which rose like a phoenix from the ashes of the now defunct nurse-led NHS Direct, has not been without criticism in the media (Wilkinson and Pickover, 2016). Despite this, Jeremy Hunt has announced that he will expand the 111 service to incorporate a new online triage service for some less serious health problems. This will help some patients to report their symptoms and receive bespoke health advice or a personal call back from a health professional, giving new opportunities for registered nurses to expand their skills. This aspect of the 111 service is being developed with leading clinicians, and the new system will be piloted to ensure the best patient outcomes.

- NHS-approved apps. A major innovation in digital technology currently being developed is the introduction of NHSapproved health apps to guide and inform patient choice. Boulos et al (2014) have estimated that as of 2012, the number of health-related apps exceeded 40000 . Furthermore, they report that many health professionals and consumers express concerns about the quality of many apps, with some lobbying for regulatory control or certification. Perhaps mindful of this,

J NHS England is scheduled to launch a library of approved NHS-assessed apps to ensure that people can select reputable and effective products to monitor their health. - Relaunching the NHS Choices website. NHS Choices was designed to illuminate patients' rights to choice in healthcare, where to find information to help choose, and how to complain if choice isn't offered. The new website will offer an improved menu of online patient services, including the ability to book GP appointments and request and monitor prescriptions. This relaunch will also see a change in name-it will soon be known as NHS.UK.

- Giving patients instant access to personal health records online. This innovation has been inspired by the 'blue button' app, used extensively in the USA, where patients can go online and securely download their own medical records (HealthIT.gov, 2016). The Government is optimistic that the new NHS.UK website will also offer a similar service by allowing patients to view and download their own medical records, giving them immediate access to vital healthcare information including prescriptions and test results. Turvey et al (2014), in a study of over 9000 blue-button users, found that the most highly endorsed benefit reported by patients was that the blue button helped them understand their health history better because all the information was in one place.

- More interactive, local information about the performance of health services. This extension of information giving was launched simultaneously with Jeremy Hunt's announcement about the Government's response to the Wachter review and is now a component of the MyNHS website (MyNHS, 2016). This website now offers improved data pertinent to the performance of the NHS across myriad services including dementia, diabetes and learning disability. The Government intends to offer maternity, cancer and mental-health data to the public in late 2016, and is planning for the website to offer maps, graphs and tools to enable patients to see more accurately how the performances of their local services have changed over time.

\section{Conclusion}

The Health and Social Care Information Centre (HSCIC) was established on 1 April 2013 and is England's trusted data source, delivering high-quality information and IT systems to help in the quest for better patient services, care and outcomes. Better known as NHS Digital (2016), its chief executive Andy Williams has endorsed the new government initiative to harness digital technology. Williams believes that this and other creative

\section{KEY POINTS}

- The Government has announced substantial funding for a range of NHS trusts to help them develop excellence in harnessing digital technology for the improvement of patient care

- The first recorded telephone consultation in the UK took place in 1879 to discount a provisional diagnosis of croup

- Telemedicine offers health professionals opportunities to address social and geographical inequalities in healthcare delivery

- The Government has a range of digital technology measures that it will develop to enhance overall patient care, including changes to the 111 service

transformations in the NHS will deliver the benefits that digital technologies will bring to doctors, nurses, social workers, patients and the public. Undoubtedly, members of the nursing profession will play a major part in this transformation. BJN

Anonymous (1879) Letter to the editor of The Lancet. 29th November 1879: 819

Boulos MNK, Brewer AC, Karimkhani C, Buller DB, Dellavalle RP (2014) Mobile medical and health apps: state of the art, concerns, regulatory control and certification. Online J Public Health Inform 5(3): 229

Department of Health (2016a) New plans to expand the use of digital technology across the NHS. http://tinyurl.com/ j96bdcl (accessed 30 September 2016)

Department of Health (2016b) Making IT work: harnessing the power of health information technology to improve care in England. http://tinyurl.com/jopv3c9 (accessed 30 September 2016)

Glasper A (1993) Telephone triage: a step forward for nursing practice? Br J Nurs 2(2): 108-9

Hasvold PE, Wootton R (2011) Use of telephone and SMS reminders to improve attendance at hospital appointments: a systematic review. J Telemed Telecare 17(7): 358-64. doi: 10.1258/jtt.2011.110707

HealthIT.gov (2016) Your Health Record. http://tinyurl. com/ztjqbg4 (accessed 30 September 2016)

May C, Ellis NT (2001) When protocols fail: technical evaluation, biomedical knowledge, and the social production of 'facts' about a telemedicine clinic. Soc Sci Med 53(8): 989-1002

MyNHS (2016) See how your CCG is performing. http:// tinyurl.com/zwl8nmv (accessed 30 September 2016)

Rosengren D, Blackwell N, Kelly G et al (1998) The use of telemedicine to treat ophthalmological emergencies in rural Australia. J Telemed Telecare 4 Suppl 1: 97-9

Turvey C, Klein D, Fix G et al (2014) Blue Button use by patients to access and share health record information using the Department of Veterans Affairs' online patient portal. J Am Med Inform Assoc 21(4): 657-63

Wachter R (2015) The Digital Doctor: Hope, Hype, and Harm at the Dawn of Medicine's Computer Age. McGraw-Hill Education, New York

Wilkinson and Pickover (2016) NHS 111 service investigated over allegations '17-year-olds answered urgent calls'. Independent online. 16 February 2016 